Documentation et bibliothèques

DOCUMENTATION BIBLIOTHEQUES

\title{
Apprendre à s'informer : les fondements et les objectifs d'une politique de formation documentaire en milieu universitaire
}

\section{Yves Tessier}

Volume 23, numéro 2, juin 1977

URI : https://id.erudit.org/iderudit/1055248ar

DOI : https://doi.org/10.7202/1055248ar

Aller au sommaire du numéro

\section{Éditeur(s)}

Association pour l'avancement des sciences et des techniques de la documentation (ASTED)

\section{ISSN}

0315-2340 (imprimé)

2291-8949 (numérique)

Découvrir la revue

\section{Citer cet article}

Tessier, Y. (1977). Apprendre à s'informer : les fondements et les objectifs d'une politique de formation documentaire en milieu universitaire. Documentation et bibliothèques, 23(2), 75-84. https://doi.org/10.7202/1055248ar
Résumé de l'article

Les changements récents dans la philosophie de l'éducation et l'évolution vers une société éducative invitent à développer davantage la dimension pédagogique intrinsèque à la définition même de bibliothèque. L'auteur élabore cette approche autour du concept de formation documentaire dont il essaie de définir les fondements, la nature, les conditions d'implantation, les objectifs et les niveaux d'application. Conçue en fonction du milieu universitaire, cette démarche conceptuelle peut également servir à alimenter la réflexion sur une dimension pédagogique applicable à d'autres milieux de bibliothèque.
Tous droits réservés (C) Association pour l'avancement des sciences et des techniques de la documentation (ASTED), 1977
Ce document est protégé par la loi sur le droit d'auteur. L'utilisation des services d'Érudit (y compris la reproduction) est assujettie à sa politique d'utilisation que vous pouvez consulter en ligne.

https://apropos.erudit.org/fr/usagers/politique-dutilisation/ 


\title{
Apprendre à s'informer: les fondements et les objectifs d'une politique de formation documentaire en milieu universitaire
}

\author{
Yves Tessier \\ Cartothèque \\ Bibliothèque de I'Université Laval \\ Québec
}

Les changements récents dans la philosophie de l'éducation et l'évolution vers une société éducative invitent à développer davantage la dimension pédagogique intrinsèque à la définition même de bibliothèque. L'auteur élabore cette approche autour du concept de formation documentaire dont il essaie de définir les fondements, la nature, les conditions d'implantation, les objectifs et les niveaux d'application. Conçue en fonction du milieu universitaire, cette démarche conceptuelle peut également servir à alimenter la réflexion sur une dimension pédagogique applicable à d'autres milieux de bibliothèque.

Recent changes in the philosophy of education and the evolution towards an educated society are an invitation to further develop the pedagogical dimension which is intrinsic to the definition of a library. The author explores this approach in the realm of user education and tries to define its basis, its nature, the conditions of its implantation, its objectives and the levels of its application. Thought out in view of university libraries, this process can also be applied to the pedagogical aspect of other types of libraries.

Frente a los cambios que sufren la filosofía de la educacion asi que la sociedad que necesita educarse siempre más, la dimension pedagógica intrínseca a la definicion de biblioteca debe redefinirse. El autor trata de definir los fundamentos de la formación documentaria, su essencja, sus condiciones de establecimiento, sus objectivos y sus niveles de aplicación. Las raíces de su reflexión se hallan 'en el medio universitario pero pueden servir de punto de partida y adaptarse a otros niveles pedagógicos $y$ por lo tanto a otros tipos de bibliotecas.

L'initiation à la bibliothèque et à ses services n'est pas un phénomène nouveau. Les traditionnelles "visites de bibliothèque" s'inscrivent dans une préoccupation naturelle pour un organisme de service d'établir des contacts avec sa clientèle afin de promouvoir auprès de cette dernière les avantages qu'il peut offrir.

Le renouvellement de la philosophie de l'éducation et l'évolution de la société, qui se veut de plus en plus éducative, projettent un éclairage nouveau sur ces activités d'initiation à la bibliothèque. De promotionnelles qu'elles ont surtout été jusqu'à maintenant, ces activités sont appelées à devenir davantage pédagogiques lorsqu'on les replace dans le contexte de l'apprentissage qui caractérise fortement les milieux actuels d'éducation.

L'intégration plus poussée de la bibliothèque aux processus d'enseignement signifie une contribution réelle et une participation plus immédiate à la formation générale des individus. Replacée dans ce 
contexte de formation, l'initiation à la bibliothèque, développée à des degrés plus poussés, rejoint le niveau de la formation documentaire des individus. Nous touchons alors à la plénitude de la dimension pédagogique inhérente à la définition des bibliothèques, surtout celles reliées directement à l'enseignement.

Exaltante, cette dimension en devenir est lourde d'implications dans sa réalisation, tant aux plans psychologique que pédagogique et administratif. Avant d'être implantée dans un milieu, cette dimension présuppose, dans son approche, un effort de réflexion axé sur les fondements qui la justifient et les objectifs que la formation documentaire doit atteindre. Ces deux éléments étant bien définis, il devient facile de déterminer par la suite les moyens à mettre en œuvre pour réaliser ces intentions. Le présent texte veut apporter, à cet effort de réflexion, une contribution surtout orientée vers le contexte universitaire. Néanmoins, elle pourra également être utile, par transposition et en y apportant les adaptations appropriées, en d'autres contextes et d'autres milieux. ${ }^{1}$

1. Ce texte reprend, dans ses grandes lignes, un document de travail préparé à l'intention du groupe d'étude mis sur pied par la Bibliothèque de l'Université Laval dans le but de préparer une politique d'ensemble de la formation documentaire. Ce groupe comprend des bibliothécaires et des conseillers du Service de pédagogie universitaire.

La documentation sur la formation documentaire est particulièrement abondante, surtout pour les années récentes. Nous voulons signaler ici trois sources d'information particulièrement utiles. Ulysse Roy, de la Bibliothèque de l'Université Laval, a compilé en juin 1975 une Bibliographie sélective sur l'initiation à la bibliothèque 19701974 comportant 270 références à des monographies, brochures, articles provenant d'une soixantaine de périodiques, et documents du système ERIC.

John Lubans $\mathrm{Jr}$ a publié un très important recueil d'études et de rapports de recherche intitulé Educating the Library User (New York, Bowker, 1974, 435 p.) Les trente-huit articles sont groupés en trois parties: "Rationale for educating library users". "Faculty involvment in library-use instruction», "Implementation and evaluation of libraryuse instruction programs». Une bibliographie sélective de douze pages complète l'ouvrage.

Le projet américain LOEX (Library orientation-ins-

\section{Fondements, définition et conditions d'implantation de la formation documentaire}

\section{L'enseignement supérieur et l'information}

L'enseignement supérieur vise à réaliser trois fonctions principales se rattachant aux connaissances: a) la transmission des connaissances actuelles, b) la découverte de connaissances nouvelles et leur intégration aux connaissances actuelles, c) l'application des connaissances à la solution de problèmes pratiques dans une société en évolution. ${ }^{2}$ L'information est présente à la base même de ces trois fonctions.

La première fonction constitue une opération de transfert d'information à l'intention d'une population parvenue à un stade avancé de formation. Ce transfert s'effectue à partir de deux types de sources d'information: les sources vivantes (professeurs et autres personnes informantes) et les sources enregistrées (documents de toutes sortes). En outre, la maîtrise de l'information est une condition essentielle à la découverte de connaissances nouvelles (recherche), si l'on ne veut pas, par mégarde intellectuelle, chercher le connu. La diffusion des connaissances nouvelles signifie également un transfert d'information à l'intention des chercheurs susceptibles d'être intéressés par ces découvertes. Enfin, l'application des connaissances à des situations concrètes fait appel au concept d'information "appliquée», laquelle

truction exchange), établi à la Eastern Michigan University, est un centre qui collige et met en circulation de l'information, de la documentation et du matériel didactique sur l'initiation à la bibliothèque sous toutes ses formes. Un bulletin d'information, LOEX News, est publié quatre fois par année. En s'inscrivant à ce centre et en acceptant d'échanger de l'information ou de la documentation sur le sujet, on peut bénéficier gratuitement des services offerts, notamment pour l'emprunt de matériel didactique. On peut communiquer avec ce centre en s'adressant à CaroIyn Kirkendall, Director, Project LOEX, Center of Educational Resources, Eastern Michigan University, Ypsilanti, Michigan 48197.

2. Voir Carl Kaysen, The Higher Learning, the Universities and the Public, Princeton, Princeton University Press, 1969, p. 52-53. 
doit être tenue à jour pour refléter l'évolution constante du milieu d'application.

Au plan des connaissances, l'information apparaît donc comme une trame sousjacente à l'enseignement supérieur et, de ce fait, représente un élément essentiel de la formation universitaire. Pour les besoins du présent propos, l'information peut être définie comme suit: un acquis de connaissances dans un but de formation ou de recherche, acquis systématique, exhaustif ou appliqué selon les trois distinctions apportées précédemment.

\section{L'apprentissage et l'information}

En plus de transmettre les connaissances actuelles et d'en faire découvrir de nouvelles, l'enseignement supérieur vise à la formation d'individus capables de rendre les services que la société est en droit d'attendre de ses membres les plus instruits. Les courants actuels de pensée dans le domaine de l'éducation tendent à centrer davantage toute formation sur l'individu, en respectant son rythme d'assimilation, ses besoins et ses intérêts. Comme l'a fait remarquer en 1972 la Commission Carnegie sur l'enseignement supérieur aux ÉtatsUnis, la transmission des connaissances, en milieu universitaire, devient comparativement secondaire en regard de l'inculcation de capacités personnelles de formation continue, particulièrement par le travail individuel et le travail en bibliothèque. ${ }^{3}$ Avec l'explosion des connaissances, la nécessité apparaît de plus en plus grande de donner aux étudiants l'occasion de développer cette aptitude à repérer et à utiliser efficacement l'information pour répondre à leurs besoins personnels ou plus tard professionnels. D'où la nécessité d'apprendre à s'informer.

La formation universitaire doit permettre à l'étudiant d'apprendre à penser, c'està-dire de développer sa capacité d'appli-

3. Carnegie Commission on Higher Education, Reform on Campus: Changing Students, Changing Academic Programs, New York, McGraw-Hill, 1972, p. 23-24. quer ses connaissances à des situations nouvelles. Mais il doit pour cela apprendre comment acquérir de nouvelles connaissances pour pouvoir en faire un usage créateur. II ne peut donc pas véritablement apprendre à penser sans apprendre à s'informer. *

Cet apprentissage des sources d'information apparaît comme une méthode de travail de plus en plus nécessaire à acquérir, si l'on veut former davantage les étudiants à solutionner eux-mêmes leurs problèmes intellectuels sans une dépendance trop grande de tuteurs. Dans le contexte de l'éducation permanente, l'étudiant "perpétuel» trouve dans la pratique systématique de l'information un moyen facilement accessible de se recycler sans cesse, en tenant à jour l'état de ses connaissances dans sa sphère d'intérêt en vue de suivre l'évolution de son milieu.

Tant au plan des connaissances qu'à celui des méthodes de travail et de recherche, l'information apparaît comme une donnée essentielle de toute formation universitaire.

\section{Définition de la formation documentaire}

La formation universitaire doit donc inclure l'apprentissage des sources enregistrées d'information, ce qui veut dire apprendre à connaître et à utiliser efficacement les ressources documentaires dans le but de répondre à des besoins personnels ou professionnels d'information.

Cet apprentissage des ressources documentaires délimite le concept de formation documentaire, qu'on peut définir ainsi: l'ensemble des activités d'apprentissage permettant de connaître et d'utiliser les ressources documentaires de façon optimale afin de répondre à des besoins d'information pour fins d'étude, de recherche et de ressourcement permanent.

4. D'après L'équipe professeur-étudiants. Sous la direction de Robley D. Evans. Traduction de Guy Godin, Québec, Université Laval, Service de pédagogie universitaire, 1973, p. 5 et 16 . (Documents, no 3). 


\section{Rôle de la bibliothèque dans la formation universitaire}

La Commission Carnegie sur l'enseignement supérieur a bien résumé le rôle pédagogique que peut jouer la bibliothèque, bien au-delà d'une certaine conception qui a prévalu trop longtemps:

"Les bibliothèques sont généralement considérées comme des centres plutôt passifs sur le campus, des endroits où des livres sont conservés et où les étudiants peuvent travailler. Elles peuvent jouer et, à certains endroits, elles jouent effectivement un rôle plus actif. Les bibliothécaires, dans les domaines de leur spécialisation, peuvent superviser des projets d'étude personnels, animer des séminaires et donner des cours sur les méthodes de recherche. Ils peuvent être considérés comme faisant partie du personnel enseignant. La bibliothèque peut devenir le centre d'utilisation de la nouvelle technologie, comme l'enseignement assisté par ordinateur et par vidéocassettes, et le centre pour l'auto-apprentissage [...] Dans bien des cas, les bibliothécaires devraient prendre une orientation plus engagée du côté de la diffusion de l'information plutôt que du côté de l'attention traditionnellement grande portée à la conservation des livres. $»^{5}$

C'est en recommandant que la bibliothèque devienne un participant plus actif au processus d'enseignement - ce qui implique une augmentation des fonds nécessaires - que la Commission Carnegie sur l'enseignement supérieur a présenté la bibliothèque comme un centre d'apprentissage à l'intérieur de l'université. Cette conception illustre un objectif hautement désirable, se rattachant à une tendance de plus en plus ressentie de mieux intégrer la bibliothèque aux processus d'enseignement et de recherche universitaires.

5. Carnegie Commission..., Reform on Campus..., 50. Traduction de l'auteur.
Dans la poursuite de cet objectif, la bibliothèque peut considérer la formation documentaire comme une manifestation privilégiée de cette volonté de réaliser l'intégration désirée à un degré poussé. II revient dès lors à la structure documentaire de l'université de promouvoir auprès des autres structures pédagogiques et administratives la maîtrise des ressources documentaires comme partie intégrante de la formation dispensée par l'université. C'est ainsi que la bibliothèque peut jouer un rôle vraiment actif dans la formation universitaire.

\section{Raisons d'être de la formation documentaire}

On peut invoquer plusieurs raisons d'ordre pratique pour justifier l'existence de la formation documentaire au niveau universitaire.

\section{Pour l'étudiant}

L'approche autonome à la solution des problèmes intellectuels des étudiants ne va pas sans un usage systématique des ressources d'information disponibles. Seule une formation spécifique en ce sens permettra cet usage à un degré fonctionnel.

L'étudiant tire davantage profit de ses études s'il peut chercher par lui-même et s'il peut élargir ses possibilités d'apprentissage par une formation documentaire appropriée venant faciliter l'autonomie personnelle dans son travail.

Dans une société submergée d'informations, l'étudiant doit acquérir une méthode qui lui permette de trouver l'information requise et de l'évaluer de façon critique.

Si l'on ne peut prouver que la réussite intellectuelle est fonction directe de la fréquentation d'une bibliothèque, il reste que les travaux actuels des étudiants s'effectuent généralement dans un contexte de sous-utilisation des bibliothèques. 


\section{Pour le professeur}

L'intégration plus poussée des sources d'information dans l'enseignement peut être l'occasion pour le professeur de renouveler ses méthodes pédagogiques dans le sens de l'évolution actuelle de l'apprentissage.

Cette intégration est de nature à améliorer la qualité et la valeur didactique des travaux personnels donnés aux étudiants et, partant, la qualité de l'enseignement dispensé.

\section{Pour le chercheur}

Le personnel de recherche qui a appris à s'informer peut réaliser avec plus d'économie et d'efficacité la partie "recherche de l'information" de tout projet de recherche.

La recherche documentée de façon systématique est susceptible d'atteindre ses fins avec un plus grand degré de réussite.

\section{Pour la bibliothèque}

La formation documentaire est susceptible de faire accroître, tant en qualité qu'en quantité, l'utilisation des ressources disponibles et d'améliorer ainsi l'efficacité générale de la bibliothèque comme service. Elle en améliore particulièrement l'efficacité au niveau du personnel qui pourra davantage œuvrer au niveau des besoins spécialisés des utilisateurs.

\section{Pour l'administration universitaire}

La formation documentaire est un concept nouveau qui permet d'améliorer la qualité de la formation universitaire aux plans des méthodes et de la préparation à une éducation continue.

Elle favorise également une meilleure gestion des ressources universitaires par une intégration plus poussée des ressources pédagogiques actuelles et des ressources considérées traditionnellement comme auxiliaires de l'enseignement et de la recherche.

Ces quelques raisons d'être de la formation documentaire laissent entrevoir de nombreux avantages qui militent en faveur de l'introduction de ce concept dans le fonctionnement normal de l'université.

\section{Principes de bases touchant à la formation documentaire}

La formation documentaire ne porte pas sur un ensemble défini de connaissances, comme une discipline, mais plutôt sur des savoir-faire intellectuels à acquérir, parmi d'autres, pour mieux atteindre les objectifs personnels d'étude et de recherche. Cet état de fait met en lumière certains principes de base à prendre en considération dans l'examen du contexte d'implantation de la formation documentaire.

1. La formation documentaire doit répondre à des préoccupations actuelles, au moment où elles sont les plus ressenties, en tenant compte du niveau des clientèles visées.

2. La formation documentaire doit être dispensée en relation étroite avec les cours ou les travaux de recherche.

3. Elle doit être axée vers des types spécifiques d'usagers et de besoins, non pas vers une clientèle-type unique, théorique et imprécise, ou seulement vers des besoins génériques.

4. Elle doit reposer sur une expérimentation personnelle de la documentation, le plus possible sur les lieux mêmes de la bibliothèque, et comporter des travaux spécifiques exigeant une évaluation.

5. Elle doit porter non seulement sur l'initiation à la bibliothèque comme telle, mais surtout sur la façon d'utiliser avec efficacité les ressources disponibles pour mieux atteindre les objectifs poursuivis.

6. La formation documentaire doit s'enraciner dans le milieu à desservir et doit nécessairement impliquer, dans son élabora- 
tion et son implantation, les professeurs qui sont le facteur le plus déterminant dans l'utilisation des ressources documentaires.

7. La formation documentaire ne doit pas être constituée d'interventions isolées mais d'activités planifiées dans une progression logique, cumulative et continue, à partir d'un niveau général ou élémentaire jusqu'à un niveau spécialisé. ${ }^{6}$

Ces quelques lignes directrices peuvent guider l'élaboration d'un programme de formation documentaire qui soit efficace, pertinent et adapté aux besoins du milieu.

\section{Conditions d'implantation de la formation documentaire}

En plus des ressources humaines et matérielles requises, certains facteurs liés au milieu doivent également être pris en considération dans l'élaboration d'un programme de formation.

\section{Les préalables}

L'idée générale prévalant sur le campus concernant la place que la bibliothèque doit occuper dans les processus d'enseignement et de recherche;

la qualité de la communication existant entre la bibliothèque, le personnel enseignant, les étudiants et la direction de l'université;

les vues de la direction de la bibliothèque et le degré de l'engagement qu'elle est prête à prendre vis-à-vis la formation documentaire;

la capacité et le désir du personnel professionnel de la bibliothèque de s'engager dans un tel programme, ainsi que l'efficacité et la motivation du personnel de soutien vis-à-vis cet engagement;

la présentation explicite de ce programme

6. Mary B. Cassata, Library Instruction Program Proposal, Buffalo, State University of New York, University Libraries, 1973, p. 2-3. (Document ERIC, no ED077541). (objectifs et contenu) et son acceptation par le milieu. ${ }^{?}$

\section{Les contraintes}

Le gigantisme et la complexité d'une grande bibliothèque;

la variété des supports d'information, leur dispersion et leurs degrés divers d'organisation;

I'hétérogénéité des besoins, des spécialités et des niveaux d'intérêt de la clientèle;

les différences, chez les usagers, dans les niveaux de connaissances préalables sur la bibliothèque;

le degré d'acceptation du bibliothécaire comme participant actif au processus d'enseignement et la démonstration de la capacité de la bibliothèque d'assumer un rôle de formation;

la disponibilité et l'attribution des ressources requises pour l'implantation valable de la formation documentaire.

\section{Les facteurs de réussite}

Motivation. La nécessité de la formation documentaire apparaît avec les problèmes d'information à surmonter. Les besoins qui en découlent motivent l'utilisateur à trouver un moyen efficace de surmonter ces problèmes. Cette motivation touche à la fois le professeur et l'étudiant.

Moment. La formation documentaire doit être dispensée au moment où elle sera perçue comme étant utile, voire nécessaire.

Pertinence. Elle ne doit comporter que la matière utilisable immédiatement ou ultérieurement (ne pas essayer de former des bibliothécaires).

Responsabilité. La formation documentai-

7. John Lubans, A Look at Library Use Instruction Programs: the Problems of Library Users and Non-users, Boulder, Colo., Colorado University Libraries, 1972, p. 5. (Document ERIC, no ED093311). 
re doit être développée en prenant en considération et en respectant le partage actuel à l'université des responsabilités à l'égard de la formation universitaire.

Initiative. Elle est mise de l'avant par la bibliothèque qui manifeste ainsi sa volonté de participer davantage à la formation dispensée par l'université.

Niveau. La formation documentaire doit être progressive, cumulative et continue, selon les niveaux des besoins à satisfaire.

Méthode. Elle doit contenir à la fois des activités d'apprentissage génériques et d'autres plus spécifiques en fonction de certains besoins précis, selon une approche qui favorise l'expérimentation personnelle et concrète de la documentation et qui respecte les préoccupations des individus.

Collaboration. La formation documentaire doit indispensablement être mise en application avec la collaboration et la participation du personnel enseignant.

Attitude pédagogique. Elle doit stimuler la fonction pédagogique des bibliothécaires et permettre de mettre en valeur les aptitudes disponibles dans ce domaine.

\section{Le milieu et la formation documentaire}

Connaît-on la perception que se font les étudiants et les professeurs des services qu'ils attendent de la bibliothèque? Sait-on ce qu'ils ont réellement besoin de connaître en matière d'information? Ces questions, alliées à la nécessité de développer des programmes pertinents, démontrent l'importance de bien connaître les besoins et les niveaux d'intérêt du milieu et de sensibiliser ce milieu à l'importance d'intégrer davantage les ressources d'information dans le fonctionnement normal de l'université.

8. John Lubans, "Objectives for library-use instruction in educational curricula" in John Lubans, ed., Educating the Library-user, New York, Bowker, 1974 , p. $217-218$.

\section{Connaissance du milieu}

Il est très important de connaître la perception qu'ont les usagers de la bibliothèque afin d'éviter de vaines tentatives de communication et aussi pour offrir une réponse qui soit des plus pertinentes en fonction des besoins ou des attentes.

II faut connaitre l'attitude et le comportement de l'usager vis-à-vis la bibliothèque et les bibliothécaires, l'usage effectif qu'il fait de la bibliothèque, sa motivation à l'utiliser ou à ne pas l'utiliser, ses raisons d'aller chercher ailleurs qu'à la bibliothèque l'information recherchée, la connaissance effective qu'il a de la bibliothèque.

Les mêmes interrogations sont particulièrement applicables aux professeurs, avec un degré de conséquence accru. En effet, le professeur est le facteur le plus déterminant dans l'exploitation des ressources documentaires car il délimite le champ d'exploration et de recherche des étudiants par les recommandations de travail qu'il leur transmet. L'inclusion systématique et intégrée de la bibliothèque dans ces recommandations entraînera nécessairement une utilisation plus grande de la bibliothèque, puisque les critères d'évaluation (découlant normalement de ces recommandations) prendront en considération le fait d'une présence efficace à la bibliothèque. ${ }^{9}$

\section{Sensibilisation du milieu}

Les avantages évidents de la formation documentaire apparaissent-ils comme tels aux yeux des principaux intéressés? Vue de l'intérieur de la bibliothèque, la formation documentaire apparaît comme une solution des plus avantageuses, favorisant une utilisation plus rationnelle des ressources documentaires existantes.

La formation documentaire est un concept non seulement à implanter, mais avant tout à répandre et à faire accepter. On peut tenter par tous les moyens de rajeunir

9. Arthur P. Young, "Research on library-user education: a review essay" in Lubans, Educating.... 2-4. 
l'image de la bibliothèque, il reste que seule la démonstration convaincante que la bibliothèque est d'une «indispensable nécessité» apparaît comme l'argumentation la plus logique à déployer pour convaincre de sa raison d'être et de son utilité. Le concept de formation documentaire s'inscrit dans ce type d'argumentation. La sensibilisation d'un milieu est une phase préparatoire très importante pour l'introduction d'un changement dans ce même milieu.

La formation documentaire apparaît ainsi comme une véritable opération de marketing: identifier et aider à définir des besoins en vue d'offrir une réponse en voie de préparation, sinon déjà prête.

\section{But, objectifs et niveaux d'application de la formation documentaire}

L'examen des fondements d'une situation a pour but de déterminer les besoins à satisfaire et les principes de base devant guider l'élaboration d'une démarche pour satisfaire ces besoins. Par la suite, la formulation des buts et des objectifs de cette démarche vient préciser les résultats à atteindre par lesquels les besoins ressentis seront satisfaits.

\section{Changements attendus}

La perception des raisons d'être et de la nécessité d'amorcer une opération d'envergure doit conduire à l'élaboration des buts et objectifs, en vue d'entreprendre des actions matérialisant l'opération envisagée. L'examen des changements souhaités et de leurs degrés possibles d'application aide à déterminer et à préciser les intentions à mettre de l'avant.

Un programme de formation vise en général à modifier les attitudes et les comportements d'individus dans un sens donné, à l'aide d'activités et à partir d'une situation initiale bien définie. Ces changements attendus se situent aux plans affectif, cognitif et psycho-moteur.

\section{Au plan affectif}

Ce type de changement permet de créer chez l'individu un climat qui favorise la réceptivité et qui facilite la réussite des interventions d'apprentissage. II vise à faire tomber les blocages psychologiques possibles qui peuvent entraver la progression dans le sens désiré de la démarche proposée.

La formation documentaire doit permettre de vaincre chez certaines clientèles les répulsions rattachées à une image non conforme de la bibliothèque, afin que cette dernière en vienne à être considérée comme un lieu agréable et valable, où l'on se rend avec intérêt pour travailler et non par obligation formelle ou morale.

\section{Au plan cognitif}

À ce niveau, il s'agit de modifier l'état des connaissances: corriger les connaissances acquises ou en fournir davantage.

La formation documentaire doit permettre de vaincre la méconnaissance (ou l'ignorance) de l'organisation et des possibilités d'exploitation des ressources documentaires et faire connaître les avantages à les utiliser.

\section{Au plan psycho-moteur}

II s'agit ici de convaincre à agir, de faire passer à l'action.

La formation documentaire doit, à ce niveau, faire en sorte que les usagers en viennent à utiliser effectivement et régulièrement les ressources d'information qu'ils connaîtront bien et qu'ils auront su intégrer à leurs activités de travail et de recherche.

Il convient de signaler que la formation documentaire doit non seulement s'étendre à la clientèle intéressée de près ou de loin, mais aussi tenter de rejoindre les non-utilisateurs pour les amener à modifier leur attitude ou leur comportement vis-à-vis les ressources documentaires. ${ }^{10}$

10. Voir J. Thomas Vogel, "A critical overview of the evaluation of library instruction». Drexel Library Quarterly, vol. 8, no. 3 (July 1972), 317-320. 


\section{But de la formation documentaire}

Un but est une déclaration générale indiquant l'orientation ou la destination vers laquelle tend un engagement quelconque.

Le but de la formation documentaire peut être ainsi formulé: fournir aux usagers l'occasion de connaître et d'utiliser efficacement les ressources documentaires disponibles pour répondre à leurs besoins d'information pendant et après leur séjour à l'université.

\section{Objectifs de la formation documentaire}

Un objectif est une communication d'intention décrivant un changement attendu à la suite d'une séquence d'activités.

On peut formuler de la manière suivante les objectifs de la formation documentaire. ${ }^{11}$

\section{Objectif général:}

développer des connaissances et des capacités individuelles de travail dans la maîtrise des ressources documentaires pour répondre à des besoins variés et continus d'information.

\section{Objectifs spécifiques:}

connaître l'existence, l'organisation et le fonctionnement général de la bibliothèque;

connaître et pouvoir utiliser des sources-types d'information en fonction de situations diverses;

être capable de cerner le champ de la documentation dans une discipline ou dans un secteur donné;

11. Lubans, aux pages 211 à 220 de son ouvrage Educating..., décrit en détail la nature d'un objectif et son contexte d'application à la formation documentaire. De plus, l'Association of College and Research Libraries a publié "Draft guidelines for bibliographic instruction in academic libraries" dans College and Research Library News, no. 11 (December 1976), 301. II s'agit d'une déclaration de principes pouvant être invoquée pour appuyer l'introduction d'un programme de formation documentaire. être capable de tenir à jour ses connaissances documentaires dans son secteur respectif;

développer des méthodes systématiques et critiques de recherche documentaire.

La détermination d'objectifs doit prendre en considération les besoins des usagers en matière de ressources documentaires. Quels peuvent être ces besoins? On peut mentionner, par exemple, la nécessité d'abord de connaître l'existence de la bibliothèque et des services qu'elle peut dispenser, de savoir en quoi elle peut être utile pour les études et la recherche, d'apprendre à s'en servir d'une manière méthodique et continue. La formulation de ces objectifs doit aussi tenir compte de la matière faisant l'objet de la formation considérée. Cette matière peut, par exemple, toucher à la description d'organismes ou de services, à la présentation de sources d'information, à la méthodologie d'évaluation des besoins d'information et des types de sources pour y répondre.

En se référant aux types de changements de comportement et d'attitude énoncés antérieurement (affectif, cognitif et psycho-moteur), on peut distinguer trois conséquences chez les utilisateurs:

- identifier la bibliothèque comme l'une des solutions premières et immédiates d'un problème ou d'un besoin d'information;

- connaître l'organisation et les modes d'utilisation des ressources documentaires disponibles;

- utiliser les ressources documentaires pour satisfaire ses besoins d'information.

\section{Niveaux d'application de la formation documentaire}

La formation documentaire doit comporter des niveaux divers d'application selon les besoins et les degrés d'intérêt de la clientèle. Comme principe servant à dis- 
tinguer ces niveaux, retenons non pas les années de présence à l'université, qui ne sont pas significatives comme telles, mais plutôt la progression du général au spécialisé dans l'état des connaissances sur les ressources d'information.

C'est ainsi qu'on peut distinguer quatre niveaux d'application de la formation documentaire. ${ }^{12}$

1. Initiation à la bibliothèque et à ses services:

connaissances élémentaires sur la localisation et le fonctionnement des divers services de la bibliothèque.

2. Initiation à la recherche en bibliothèque:

connaissance des sources d'information de base dans une discipline ou un secteur d'étude donné.

3. Introduction à la documentation spécialisée:

connaissance d'un champ spécifique de documentation et méthodes de travail.

4. Techniques de recherche documentaire:

maîtrise d'un champ donné de documentation et méthodologie de la recherche documentaire et du repérage de l'information.

La détermination précise des objectifs et des niveaux d'application de la formation documentaire facilite grandement l'élaboration des programmes à mettre en œuvre pour réaliser les intentions poursuivies. Car s'il faut réfléchir sur les actions à entreprendre, il faut également s'ingénier à trouver les moyens les plus efficaces de passer à l'action. L' "opérationnalisation» de la formation documentaire suggère une tout autre réflexion pas moins élaborée... qu'il nous faut maintenant entreprendre.

\section{Conclusion}

L'étude des fondements et des objectifs d'un projet d'envergure à caractère pédagogique constitue le point de départ de l'élaboration d'une politique d'ensemble. II faut également examiner les autres composantes, toutes aussi essentielles, de cette opération de planification. Les programmes viennent définir les cadres de travail à l'intérieur desquels les ressources humaines et matérielles servent à déterminer les moyens à mettre en œuvre pour atteindre les objectifs. Cette atteinte est mesurée par une évaluation appropriée; la rétroaction qui en découle permet d'améliorer l'ensemble de la stratégie.

La grille conceptuelle étant déjà tracée, il reste à en noircir les espaces significatifs pour révéler le motif de l'œuvre à construire. 